\title{
Interferência da intensidade da atividade aquática nos níveis de pressão arterial em mulheres adultas e idosas
}

\author{
Interference of intensity of aquatic activity levels in blood pressure in \\ adult and old women
}

\author{
Daniela Virote Kassick Müller ${ }^{1}$, Anne Brandolt Larrè̀2, Sabine Possa Marroni ${ }^{3}$ \\ ${ }^{1}$ Fisioterapeuta. Mestre em Engenharia de Produção com ênfase em Ergonomia pela Universidade Federal do Rio Grande do Sul (UFRGS). \\ Doutoranda do Programa de Pós-Graduação em Gerontologia Biomédica da Pontifícia Universidade Católica do Rio Grande do Sul (PUCRS). \\ Professora da Universidade Regional de Blumenau (FURB). Blumenau, SC. \\ 2 Farmacêutica bioquímica. Mestranda do Programa de Pós-Graduação em Gerontologia Biomédica da PUCRS. Porto Alegre, RS. \\ ${ }_{3}^{3}$ Psicóloga. Doutoranda do Programa de Pós-Graduação em Gerontologia Biomédica da PUCRS. Porto Alegre, RS.
}

\section{RESUMO}

Objetivos: Comparar os efeitos de programas de treinamento aeróbico e resistido com atividade aquática moderada e vigorosa nos níveis de pressão arterial em mulheres adultas e idosas normotensas ou hipertensas controladas sem medicação.

Métodos: A amostra foi intencional, constituindo-se o Grupo 1 de 9 mulheres, com idade média de 54,56 $\pm 7,04$ anos, que realizavam atividade aquática moderada $(60-70 \%$ da frequência cardíaca máxima) e o Grupo 2 de 11 mulheres com idade média de 54,91 7 ,70 anos, que realizavam atividade aquática vigorosa $(70-80 \%$ da frequência cardíaca máxima). Os dois grupos foram analisados quanto à pressão arterial durante um período de dois meses e meio de tratamento, totalizando 20 atendimentos para cada grupo. As sessões ocorriam com frequência de duas vezes por semana, com duração de 50 minutos cada aula. A intensidade do exercício era estabelecida conforme a frequência cardíaca máxima de cada indivíduo. A análise dos resultados foi feita por estatística inferencial utilizando o teste t de Student para amostras independentes e pareadas, com significância menor que 5\%.

Resultados: O Grupo 1 obteve uma diferença média intergrupo de pressão arterial sistêmica entre o início e o final do estudo de $-5,56 \mathrm{mmHg}$, enquanto o Grupo 2 apresentou diferença de $+6,91(p<0,001)$. Em relação à pressão arterial diastólica, a diferença média intergrupo do Grupo 1 foi de $-3,50$ e para o Grupo 2 de $+3,18(p=0,011)$.

Conclusões: A atividade aquática moderada foi mais eficiente que a vigorosa para a redução dos níveis pressóricos em mulheres adultas e idosas.

DESCRITORES: ATIVIDADE AQUÁTICA; PRESSÃO SANGUÍNEA; PRESSÃO ARTERIAL; PRESSÃO SISTÓLICA; PRESSÃO DIASTÓLICA; MULHERES ADULTAS; MULHERES IDOSAS.

\begin{abstract}
Aims: To compare the effects of aerobic training programs and resistance to aquatic moderate and vigorous activity in blood pressure levels in adult and elderly women, normotensive or hypertensive controlled without medication.

Methods: Sample was intentional, with Group 1 constituted of 9 women, mean age 54.56 \pm 7.04 years, who underwent moderate aquatic activity (60-70\% of maximum heart rate) and Group 2 constituted of 11 women with mean age $54.91 \pm 7.70$ years, who performed vigorous aquatic activity (70-80\% of maximum heart rate). The two groups were analyzed for blood pressure over a period of $21 / 2$ months of treatment, a total of 20 attendances for each group. The sessions occurred with a frequency of twice a week, lasting 50 minutes each class. Exercise intensity was set as the maximum heart rate of each individual. Analysis was made by inferential statistics using Student $t$ test for independent and paired samples, with significance lower than $5 \%$

Results: Group 1 had a mean difference of intergroup blood pressure, between the beginning and end of the study, of $-5.56 \mathrm{mmHg}$, whereas Group 2 showed a difference of $+6.91(p<0.001)$. In relation to diastolic blood pressure, the average intergroup difference of group 1 was -3.50 and for group was $2+3.18(p=0.011)$.

Conclusions: Moderate water activity was more efficient than vigorous water activity for reduction of blood pressure in adults and elderly women.
\end{abstract}

KEY WORDS: AQUATIC ACTIVITY; BLOOD PRESSURE; ARTERIAL PRESSURE; SYSTOLIC PRESSURE; DIASTOLIC PRESSURE; ADULT FEMALES; OLDER WOMEN. 


\section{INTRODUÇÃO}

A população idosa vem crescendo em nosso país, assim como em todo o mundo, devido ao aumento da expectativa de vida; consequentemente, cresce o número de pessoas que ultrapassa a barreira dos 60 anos de idade. No entanto, o envelhecimento é um processo de degradação progressiva, sendo impossível datar seu início, porque de acordo com o nível em que ele se situa (biológico, psicológico ou sociológico), sua velocidade e gravidade variam de indivíduo para indivíduo. Dessa forma, o processo de envelhecimento não deve ser datado como terceira ou quarta idade, devendo ser estudado entre crianças, adultos e idosos propriamente ditos. ${ }^{1}$

Uma das características do processo de envelhecimento é o aumento da incidência de doenças relacionadas a esse período de vida, ${ }^{2-4}$ destacando-se as cardiovasculares, segundo a Organização Mundial da Saúde. ${ }^{5}$ Além disso, outros fatores de risco podem estar presentes, como hipertensão, diabetes mellitus, fumo, dislipidemias, sedentarismo e obesidade, prejudicando ainda mais a saúde do idoso. A prevalência de hipertensão arterial sistêmica (HAS) chega a 60\% nessa população. ${ }^{6} \mathrm{O}$ aumento da pressão arterial (PA) resulta de modificações estruturais e funcionais no coração e nos vasos, além de alterações no sistema nervoso autônomo. ${ }^{7,8}$

Estar engajado em programas de exercícios regulares proporciona aumento da força muscular, melhora do condicionamento cardiorrespiratório, redução de gordura, aumento da densidade mineral óssea, recuperação de aspectos emocionais e melhora da qualidade de vida. ${ }^{9,10}$ A prática regular de atividade física promove melhora do controle da PA. Assim, indivíduos praticantes de exercício físico possuem 30\% menos chance de desenvolverem HAS quando comparados com seus semelhantes sedentários. Alguns autores referem que o efeito de reduzir a PA evidencia-se em indivíduos que praticam atividades vigorosas. ${ }^{11}$ Entretanto, a literatura confirma que no cenário da função cardiovascular, os exercícios aeróbicos se destacam como importante intervenção para a prevenção de doenças, devido a diversos mecanismos, como o processo de vasodilatação periférica, que auxiliam na manutenção da PA em níveis adequados, redução do tônus simpático no coração, que promove bradicardia de repouso, melhora da sensibilidade barorreflexa, dentre outros..$^{2,11,12}$ Os autores ainda evidenciam que, em relação a estes exercícios, em normotensos, os efeitos hipotensores são potencializados com a execução de exercícios de intensidade leve a moderada e duração prolongada, executados várias vezes por semana..$^{13}$
Em contrapartida, é bastante discutido que o exercício resistido promove aumento exacerbado da PA durante sua execução, tanto em indivíduos normotensos quanto em hipertensos. Este fato poderia contribuir para um aumento de risco na população hipertensa. ${ }^{14,15}$ No entanto, normalmente são indicados exercícios resistidos para o público idoso, que frequentemente apresenta HAS, porque os mesmos promovem aumento ou manutenção de massa, potência e força muscular, as quais tendem a diminuir a partir da sexta década de vida, além de contribuir na manutenção da densidade mineral óssea. ${ }^{16}$

Sendo assim, hoje em dia algumas diretrizes, como a da American Heart Association, passaram a recomendar o treinamento resistido ou de força na complementação do treinamento aeróbico para indivíduos com distúrbios cardiovasculares, sobretudo mulheres e idosos. Essas recomendações de baseiam nos comprovados efeitos benéficos que o exercício resistido promove sobre o sistema osteomuscular, assim como a indícios de que o mesmo atua de forma positiva sobre alguns fatores de risco cardiovascular. ${ }^{17}$

Entretanto, nem todos os exercícios são adequados para pessoas com problemas cardiovasculares, principalmente sendo estas idosas. Considera-se que os exercícios aquáticos são indicados por exercerem um efeito de reequilíbrio da PA. Porém, ainda existem poucos estudos científicos que relacionam a interferência da atividade aquática nos níveis de PA e poucas pesquisas que avaliam a intensidade de exercícios mais vigorosos entre hipertensos. Por esta razão, o objetivo do estudo foi comparar os efeitos da atividade aquática moderada e vigorosa nos níveis de PA em mulheres adultas e idosas.

\section{MÉTODOS}

O estudo consistiu em uma coorte concorrente com população fechada. A amostra foi intencional, constituída por mulheres participantes do programa de extensão Programa de Educação Permanente (PROEP), vinculado à Universidade Regional de Blumenau (FURB). Esse programa é composto por um total de 216 integrantes, com idade a partir dos 45 anos, que praticam diferentes atividades físicas, sociais e culturais, em diferentes projetos de extensão. Dentre os projetos de extensão pertencentes ao PROEP, destaca-se o projeto intitulado "Atividade aquática para a terceira idade", que objetiva a promoção de um envelhecimento ativo e saudável para os participantes. Esse projeto ocorre na FURB desde o segundo semestre de 2006 e, desde então, são feitas coletas de 
dados entre os participantes para o desenvolvimento de pesquisas e trabalhos de conclusão de curso. Com isto, selecionaram-se duas coletas com grupos de indivíduos semelhantes, em que foram analisados os mesmos parâmetros, da mesma forma, porém em momentos diferentes. Dentre as coletas de dados, selecionaram-se as amostras conforme os seguintes critérios de inclusão: sexo feminino, idade a partir de 45 anos, normotensas ou HAS controlada que não fizessem uso de medicação, deambuladoras, sem contraindicações à imersão no meio líquido e que apresentassem a frequência esperada para a coleta. Desta forma, formou-se o Grupo 1, com 9 mulheres, e o Grupo 2, com 11 mulheres, todas com idade compreendida entre 46 e 69 anos.

Como instrumentos de pesquisa foram utilizados um estetoscópio da marca Rappaport Premium e um esfigmomanômetro aneroide da marca Solidor. O primeiro grupo (Grupo 1) teve sua coleta de dados realizada no primeiro semestre de 2010. Esse grupo realizava atividade aquática com intensidade moderada. O segundo grupo (Grupo 2) teve seus dados coletados no primeiro semestre de 2012. O Grupo 2 realizava atividade aquática vigorosa. Ambos os grupos foram analisados durante um período de dois meses e meio, realizando 20 atendimentos no total cada grupo, com frequência de duas vezes semanais, sendo que a aferição da PA era feita uma vez por semana para posterior comparação.

A PA foi mensurada antes e após as sessões de atividade aquática, para ambos os grupos, durante 10 dos 20 encontros, sempre uma vez por semana, no mesmo dia (quarta-feira), sendo estabelecidas a PA de repouso e a PA pós atividade. Para a verificação da PA de repouso, todos os indivíduos permaneciam, previamente à aferição, cinco minutos sentados confortavelmente, em local calmo e silencioso, com o braço apoiado ao nível do coração. A aferição, por convenção, sempre foi executada no membro superior direito. A PA foi aferida sobre a artéria braquial, baseando-se nas recomendações da Sociedade Brasileira de Cardiologia. ${ }^{6}$

O Grupo 1 foi submetido a atividades aquáticas de moderada intensidade, ou seja, com frequência cardíaca (FC) mantida entre 60 a $70 \%$ da frequência cardíaca máxima (FCM); já o Grupo 2 foi submetido a atividades mais vigorosas, com FCM em torno de 70 a $80 \%$. A FCM era calculada de acordo com a fórmula de Karvonen, ${ }^{18}$ por ser uma técnica simples e de fácil reprodução, sendo mensurada antes da aplicação do protocolo junto com o levantamento do perfil sociodemográfico dos sujeitos. Para a manutenção da intensidade de exercícios dentro de cada protocolo, durante os atendimentos, era mensurada a FC para avaliar se o ritmo de trabalho permanecia adequado aos sujeitos.

Para a pesquisa, a água da piscina era mantida a uma temperatura entre $33^{\circ}$ e $35^{\circ} \mathrm{C}^{19,20} \mathrm{e}$ cada sessão tinha duração de 50 minutos, sendo realizados exercícios aeróbicos (caminhadas e corridas na água, pedaladas, exercícios ativos contra a turbulência, entre outros), com duração entre 10 e 15 minutos; exercícios resistidos de fortalecimento para tronco, membros superiores e inferiores (exercícios que exploravam a resistência contra o empuxo e turbulência, com utilização de flutuadores e implementos com aumento de área frontal), durante 20 a 30 minutos; e, por fim, atividades que desenvolviam treino de equilíbrio e coordenação, bem como atividades lúdicas e de relaxamento e flexibilização. As atividades eram as mesmas tanto para o Grupo 1 quanto para o Grupo 2. A variação estava na intensidade dos exercícios: atividades moderadas $(60-70 \%$ FCM) e vigorosas (70-80\% FCM). Os objetivos do programa de treinamento eram o aprimoramento da capacidade cardiorrespiratória e a promoção de ganho de força muscular, resistência muscular localizada, flexibilidade e amplitude de movimento articular. Todas as atividades propostas eram realizadas pela fisioterapeuta responsável pelo projeto.

A variável pressão arterial foi analisada de forma quantitativa por meio de estatística inferencial através de teste t de Student para amostras independentes e pareadas, considerando-se valores significativos aqueles com $\mathrm{p}$ menor que 0,05 . O pacote estatístico utilizado foi o SPSS versão 13. A pesquisa foi aprovada pelo Comitê de Ética da Universidade Regional de Blumenau sob o protocolo de $n^{\circ} 033 / 10$ e todos os integrantes assinaram o termo de consentimento livre e esclarecido para participar da mesma.

\section{RESULTADOS}

O Grupo 1, que realizou atividade aquática moderada, compreendia nove mulheres com média de idade de 54,56 77,04 anos. A menor idade apresentada pelo Grupo 1 foi de 46 anos, enquanto a maior idade foi de 63 anos. Já o Grupo 2, composto por 11 mulheres que realizavam atividade aquática vigorosa,

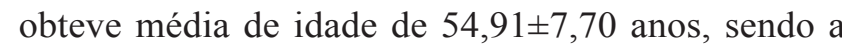
menor idade 47 anos e a maior 69 anos. A Tabela 1 analisa a variação da média de pressão arterial sistólica (PAS) e pressão arterial diastólica (PAD) nas representantes dos dois grupos. Seguindo os critérios de inclusão, nenhuma das mulheres relatava fazer uso de medicação para controle da pressão arterial, 
todas negavam tabagismo e relatavam que o uso de álcool era esporádico, todas eram socialmente ativas e $80 \%$ ainda realizavam atividades remuneradas. A maioria das mulheres analisadas eram casadas, sendo que apenas três eram viúvas e uma divorciada. Todas apresentavam apenas a atividade na piscina como prática regular de exercícios, com tempo mínimo de execução de dois e máximo de quatro meses. Em relação ao índice de massa corporal (IMC), 40\% da amostra apresentava massa corporal dentro dos valores considerados normais, $43 \%$ apresentava sobrepeso e 17\% obesidade (Tabela 1).

Houve uma tendência de diminuição nos níveis da PAS e PAD para o Grupo 1 (análise intragrupo) que, anteriormente às intervenções, apresentava uma média geral de PAS de 124,22 $\mathrm{mmHg}$ e passou para 118,67 mmHg e de pressão PAD que passou de $77,83 \mathrm{mmHg}$ para $74,33 \mathrm{mmHg}$. Estes resultados mostraram-se estatisticamente significativos no teste $\mathrm{t}$ de Student pareado tanto para a PAS $(p=0,001)$, quanto para a PAD $(p=0,043)$. No Grupo 2, é possível perceber que tanto a PAS quanto a PAD tenderam a aumentar após as intervenções, passando a PAS de uma média geral de $132,91 \mathrm{mmHg}$ para $139,82 \mathrm{mmHg}$ e a PAD de $85,27 \mathrm{mmHg}$ para $88,45 \mathrm{mmHg}$. No entanto, pelo teste $\mathrm{t}$ de Student pareado observou-se resultado significativo apenas para o aumento da PAS $(p=0,006)$. A Tabela 2 faz a comparação entre as diferenças médias da PAS e PAD (depois e antes) da atividade aquática nos dois grupos utilizando o teste t de Student para amostras independentes (análise intergrupo).

Os resultados apontam que o Grupo 1 obteve uma diferença média de PAS de menos 5,56 mmHg, ou seja, houve diminuição, enquanto o Grupo 2 apresentou mais 6,91 , mostrando aumento ao final do período de tratamento. Em relação à PAD a diferença média do Grupo 1 foi de $-3,50$ e para o Grupo 2 de $+3,18$, também representando que esta variação média aumentou após as intervenções. Pela análise estatística percebe-se que a diferença média tanto da PAS quanto da PAD foram significativas, já que a primeira apresentou valor de $p<0,001$ e a segunda $p=0,011$.

Tabela 1. Variação da média de pressão arterial sistólica e pressão arterial diastólica em dois grupos de mulheres adultas e idosas, antes e depois da participação em um programa de atividade aquática. Blumenau, Santa Catarina, 2012. Grupo 1 (G1): atividade aquática moderada (60-70\% da frequência cardíaca máxima); Grupo 2 (G2): atividade aquática vigorosa (70-80\% da frequência cardíaca máxima).

\begin{tabular}{ccccccc}
\hline \multirow{2}{*}{ Pressão arterial } & \multirow{2}{*}{} & \multicolumn{2}{c}{ Antes } & \multicolumn{2}{c}{ Depois } & \multirow{2}{*}{ Valor de $\boldsymbol{p}$} \\
\cline { 3 - 6 } & & Média & Desvio Padrão & Média & Desvio Padrão & \\
\hline PAS média G1 & 9 & 124,22 & 17,82 & 118,67 & 16,02 & $0,001^{*}$ \\
PAD média G1 & 9 & 77,83 & 9,73 & 74,33 & 10,31 & $0,043^{*}$ \\
PAS média G2 & 11 & 132,91 & 14,61 & 139,82 & 15,71 & $0,006^{*}$ \\
PAD média G2 & 11 & 85,27 & 9,46 & 88,45 & 6,88 & 0,102 \\
\hline
\end{tabular}

* Significativo. Teste t de Student.

PAS: pressão arterial sistólica; PAD: pressão arterial diastólica.

Tabela 2. Comparação entre as diferenças médias da pressão arterial sistólica e pressão arterial diastólica de dois grupos de mulheres adultas e idosas, após a participação em um programa de atividade aquática. Blumenau, Santa Catarina, 2012. Grupo 1 (G1): atividade aquática moderada (60-70\% da frequência cardíaca máxima); Grupo 2 (G2): atividade aquática vigorosa (70-80\% da frequência cardíaca máxima).

\begin{tabular}{ccccccc}
\hline Pressão arterial & Grupo & $\mathbf{n}$ & Média & Desvio Padrão & $\begin{array}{c}\text { Intervalo de } \\
\text { Confiança }\end{array}$ & Valor de $\boldsymbol{p}$ \\
\hline \multirow{2}{*}{ PAS média } & G1 - moderada & 9 & $-5,56$ & 3,32 & $-8,11$ a $-3,00$ & $0,000^{*}$ \\
& G2 - vigorosa & 11 & 6,91 & 6,67 & 2,42 a 11,39 & \\
\multirow{2}{*}{ PAD média } & G1 - moderada & 9 & $-3,5$ & 4,38 & $-6,86$ a $-0,13$ & $0,011^{*}$ \\
& G2 - vigorosa & 11 & 3,18 & 5,86 & $-0,75$ a 7,11 & \\
\hline
\end{tabular}

* Significativo. Teste t de Student.

PAS: pressão arterial sistólica; PAD: pressão arterial diastólica. 
Tabela 3. Caracterização da amostra segundo idade e pressão arterial sistólica e diastólica, no momento de avaliação para o estudo. Blumenau, Santa Catarina, 2012.

\begin{tabular}{cccc}
\hline & Idade & PAS Inicial & PAD Inicial \\
\hline Grupo 1 & & & \\
1 & 48 & 160 & 100 \\
2 & 63 & 120 & 80 \\
3 & 54 & 100 & 60 \\
4 & 50 & 160 & 100 \\
5 & 63 & 120 & 80 \\
6 & 46 & 140 & 80 \\
7 & 56 & 150 & 80 \\
8 & 48 & 120 & 80 \\
9 & 63 & 120 & 80 \\
Grupo 2 & & & \\
1 & 59 & 180 & 150 \\
2 & 52 & 160 & 100 \\
3 & 54 & 150 & 100 \\
4 & 47 & 120 & 80 \\
5 & 50 & 130 & 80 \\
6 & 49 & 100 & 70 \\
7 & 61 & 140 & 100 \\
8 & 50 & 130 & 80 \\
9 & 69 & 90 & 80 \\
10 & 66 & 150 & 100 \\
11 & 47 & 130 & 80 \\
\hline
\end{tabular}

PAS: pressão arterial sistólica; PAD: pressão arterial diastólica.

\section{DISCUSSÃO}

Este estudo confirma pesquisas anteriores que comprovam o efeito favorável do exercício dinâmico, de leve a moderada intensidade, na redução da PA em hipertensos. ${ }^{21-24}$ Monteiro et al. ${ }^{22}$ verificaram que exercícios físicos aeróbicos associados a um tratamento farmacológico são eficientes para melhorar a capacidade cardiorrespiratória, reduzindo, assim, a PA desses indivíduos. Este fato pode estar associado ao uso de grandes grupos musculares nos exercícios, como atividades de caminhada, pedalada, corrida ou natação, uma vez que possibilitam um ajuste cardiovascular devido ao aumento da resistência e da força musculoesquelética. ${ }^{22}$

Neste estudo também se observou a diminuição dos níveis de PA com exercícios aeróbicos e resistidos, mesmo sem associação medicamentosa. Porém, essa diminuição estava vinculada mais à intensidade dos exercícios do que ao tipo de exercício, uma vez que ocorreu nas mulheres que realizaram atividade de moderada intensidade, não sendo observado o mesmo naquelas que praticavam exercícios de intensidade vigorosa.
Segundo Queiroz et al. ${ }^{17}$ os exercícios resistidos também podem ter efeito hipotensor, principalmente em indivíduos normotensos e com o treinamento de baixa intensidade, o que vai ao encontro dos achados desta pesquisa. É importante ressaltar que esses autores não observaram aumento de PA nem em hipertensos nem em normotensos, enquanto neste estudo verificouse aumento da PA nas mulheres que executaram atividades físicas vigorosas. Outra condição que influencia o sistema cardiovascular e norteia a queda pressórica seriam as adaptações, referentes aos efeitos crônicos do exercício físico, resultando em modificações autonômicas e hemodinâmicas. ${ }^{21}$

Em relação ao tempo de atividade física, Monteiro et al. ${ }^{22}$ demonstraram que quatro meses de exercício regular foram eficientes para reduzir a PAS de pacientes hipertensos. A redução foi mais expressiva após o terceiro mês e não foi associada com a diminuição da pressão arterial média (PAM) e da PAD, o que sugere que um período mais longo de exercício poderia alterar efetivamente a PA. Ainda é importante ressaltar que em sua pesquisa os níveis de PA não foram muito altos no início do programa de exercícios, uma vez que os pacientes hipertensos estavam sob tratamento farmacológico. ${ }^{22}$ Nos resultados deste estudo constatouse diminuição tanto dos níveis de PAS quanto PAD em atividades aeróbicas e de resistência com intensidade moderada. No entanto, os indivíduos avaliados eram tanto normotensos quanto hipertensos, mas sem intervenção medicamentosa associada, o que sugere que a atividade física moderada é bem indicada no tratamento da HAS, mesmo em períodos menores de quatro meses.

Corroborando com os achados desta pesquisa, Barroso et al. ${ }^{23}$ descrevem uma redução discreta ou neutra da PA, principalmente após a décima semana de atividade física regular. Os mesmos autores concluem ainda que programas de atividade física supervisionada podem ser úteis na implementação de tratamento não farmacológico de HAS, associando exercícios aeróbicos com resistidos. ${ }^{23}$ A prevenção e o tratamento da hipertensão através de intervenções não medicamentosas vem crescendo e conquistando adeptos, profissionais da saúde e pacientes, que utilizam cada vez mais esta conduta terapêutica, desfrutando dos seus benefícios a médio e longo prazo.

Apesar das limitações no presente estudo, tais como número amostral pequeno, faixas etárias distintas (mulheres adultas e idosas) e tempos diferentes para coleta de dados, mesmo seguindo-se critérios metodológicos similares em ambas as aquisições, os resultados apontam que a atividade aquática contribui para a diminuição da PA, sendo que a moderada foi 
mais eficiente que a vigorosa para a redução dos níveis pressóricos na amostra estudada. Embora ainda seja controversa na literatura vigente a melhor recomendação de exercício acerca de quantidade, tipo e intensidade, os resultados deste estudo inferem que a atividade aquática moderada é melhor indicada do que a vigorosa para redução dos níveis de PA em mulheres adultas e idosas.

\section{REFERÊNCIAS}

1. Cancela DMG. O processo de envelhecimento. Psicologia. pt: o portal dos psicólogos. [Internet]. [citado 2013 ago 10]. Disponível em: http://www.psicologia.pt/artigos/ver artigo_licenciatura.php?codigo=TL0097

2. Matsudo S, Matsudo VKR. Prescrição e benefícios da atividade física na terceira idade. Rev Bras Cienc Mov. 1992;5(4):19-28.

3. Gandolfi LM, Skora MC. Fisioterapia preventiva em grupos na terceira idade. Fisioter Mov. 2001;XIII(2):55-62.

4. Ruwer SL, Rossi AG, Simon LF. Equilíbrio no idoso. Rev Bras Otorrinolaringol. 2005;71( 3):300-4.

5. World Health Organization. Cardiovascular Disease. [Internet]. [cited 2010 fev 2]. Available from: http://www. who.int/cardiovascular_diseases/en/index.html

6. Sociedade Brasileira de Cardiologia. Sociedade Brasileira de Hipertensão. Sociedade Brasileira de Nefrologia. V Diretrizes brasileiras de hipertensão arterial. Hipertensão. 2006;9(4):121-56.

7. Zallavsky C, Gus I. Idoso: doença cardíaca e comorbidades. Arq Bras Cardiol. 2002;79(6):635-9.

8. Cheitlin MD. Cardiovascular physiology: changes with aging. Am J Geriatr Cardiol. 2003;12(1):9-13.

9. Matsudo S, Matsudo VKR, Barros Neto TL. Impacto do envelhecimento nas variáveis antropométricas, neuromotoras e metabólicas da aptidão física. Rev Bras Cienc Movim. 2000;4:21-32.

10. Salles-Costa R, Heiborn ML, Werneck GL, Faerstein E, Lopes CS. Gênero e prática de atividade física e lazer. Cad Saúde Publica. 2003;19(2):325-33.

11. Fagard RH. Physical activity physical fitness and the incidence of hipertension. J Hypertens. 2005;23(2):265-67.
12. Lobo FS, Medina FL, Forjaz CLM. Efeito hipotensor do exercício físico. Hipertensão. 2010:101-7.

13. Sale DG, Moroz DE, McKelvie RS, MacDougall JD, McCartney N. Effect of training on the blood pressure response to weight lifting. J Appl Physiol. 1994;19(1): 60-74.

14. McCartney N, McKelvie RS, Martin J, Sale DG, MacDougall JD. Weight-training-induced attenuation of the circulatory response o folder males to weight lifting. J Appl Physiol. 1993;74(3):1056-60.

15. Moreland JD, Richardson JA, Goldsmith CH, Clase CM. Muscle weakness and falls in older adults: a systematic review and meta-analysis. J Am Geriatr Soc. 2004;52(7):1121-9.

16. Reeves ND, Narici MV, Maganaris CN. Myotendinous plasticity to ageing and resistance exercise in humans. Exp Physiol. 2006;91(3):483-98.

17. Queiroz AC, Kanegusuku H, Forjaz CLM. Efeitos do treinamento resistido sobre a pressão arterial de idosos. Arq Bras Cardiol. 2010;95(1):35-40.

18. Karvonen JJ, Kentala E, Mustala O. The effects of training on heart rate: a longitudinal study. Ann Med Exp Biol Fenn. 1957;35:307-15.

19. Carregaro R1, Toledo AM. Efeitos fisiológicos e evidências científicas da eficácia da fisioterapia aquática. Rev Movimento. 2008;1(1):23-7.

20. Candeloro JM, Caromano FA. Fundamentos da hidroterapia para idosos. Arq Cienc Saúde Unipar. 2001;5(2):187-95.

21. Mazo GZ, Lopes MA, Benedetti TB. Atividade física e o idoso: concepção gerontologica. $2^{\mathrm{a}}$ ed. Porto Alegre: Sulina; 2004.

22. Monteiro HL, Rolim LMC, Squinca DA, Silva FC, Ticianeli CCC, Amaral SL. Efetividade de um programa de exercícios no condicionamento físico, perfil metabólico e pressão arterial de pacientes hipertensos. Rev Bras Med Sport. 2007;13(2):107-12.

23. Barroso WKS, Jardim PCBV, Vitorino PV, Bitencourt A, Miquetichuc F. Influência da atividade física programada na pressão arterial de idosos hipertensos sob tratamento não farmacológico. Rev Assoc Med Bras. 2008;54(4): 328-33.

24. Rego ARON, Gomes ALM, Veras RP, Junior EDA, Alkimin RMN, Dantas EHM. Pressão arterial após programa de exercício físico supervisionado em mulheres idosas hipertensas. Rev Bras Med Sport. 2011;17(5):300-4 